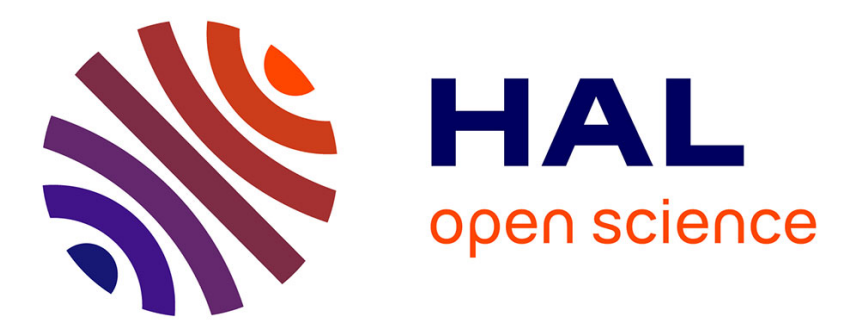

\title{
Domain-related origin of magnetic relaxation in compressively strained manganite thin films
}

S R Bakaul, B F Miao, W Lin, W. Hu, A David, H. F. Ding, T. Wu

\section{To cite this version:}

S R Bakaul, B F Miao, W Lin, W. Hu, A David, et al.. Domain-related origin of magnetic relaxation in compressively strained manganite thin films. Applied Physics Letters, 2012, 101, pp.012408. 10.1063/1.4733320 . hal-02162385

\section{HAL Id: hal-02162385 \\ https://hal.science/hal-02162385}

Submitted on 21 Jun 2019

HAL is a multi-disciplinary open access archive for the deposit and dissemination of scientific research documents, whether they are published or not. The documents may come from teaching and research institutions in France or abroad, or from public or private research centers.
L'archive ouverte pluridisciplinaire HAL, est destinée au dépôt et à la diffusion de documents scientifiques de niveau recherche, publiés ou non, émanant des établissements d'enseignement et de recherche français ou étrangers, des laboratoires publics ou privés. 


\section{AIP Applied Physics \\ Letters}

\section{Domain-related origin of magnetic relaxation in compressively strained manganite thin films}

S. R. Bakaul, B. F. Miao, W. Lin, W. Hu, A. David et al.

Citation: Appl. Phys. Lett. 101, 012408 (2012); doi: 10.1063/1.4733320

View online: http://dx.doi.org/10.1063/1.4733320

View Table of Contents: http://apl.aip.org/resource/1/APPLAB/v101/i1

Published by the American Institute of Physics.

\section{Additional information on Appl. Phys. Lett.}

Journal Homepage: http://apl.aip.org/

Journal Information: http://apl.aip.org/about/about_the_journal

Top downloads: http://apl.aip.org/features/most_downloaded

Information for Authors: http://apl.aip.org/authors

\section{ADVERTISEMENT}

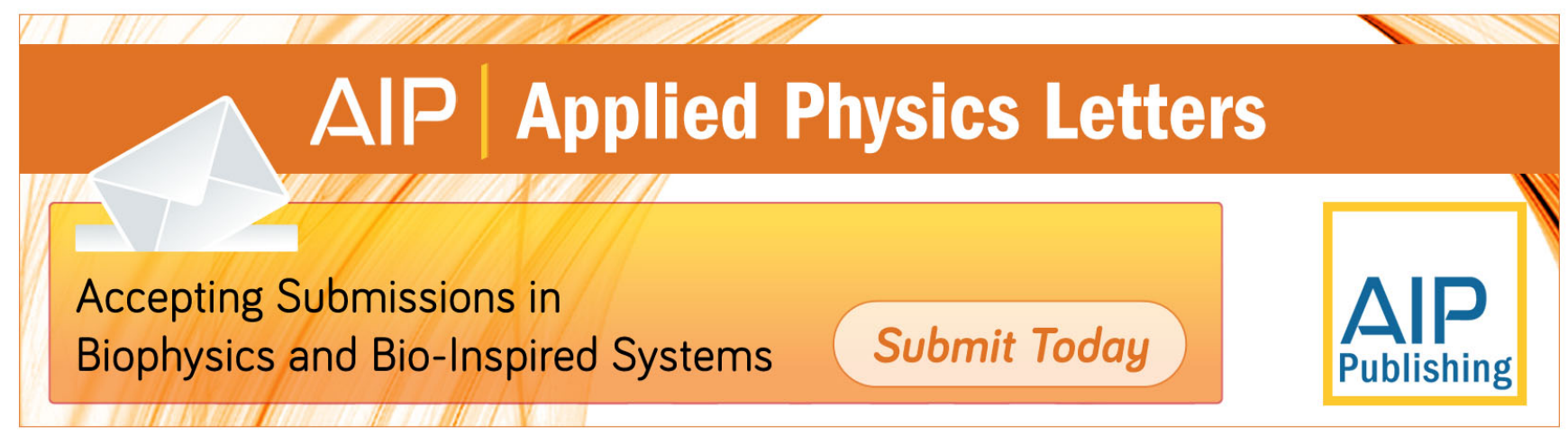




\title{
Domain-related origin of magnetic relaxation in compressively strained manganite thin films
}

\author{
S. R. Bakaul, ${ }^{1}$ B. F. Miao, ${ }^{2}$ W. Lin,${ }^{1}$ W. Hu, ${ }^{1}$ A. David, ${ }^{1}$ H. F. Ding,${ }^{2}$ and T. Wu ${ }^{1, a)}$ \\ ${ }^{1}$ Division of Physics and Applied Physics, School of Physical and Mathematical Sciences, \\ Nanyang Technological University, Singapore 637371, Singapore \\ ${ }^{2}$ National Laboratory of Solid State Microstructures and Department of Physics, Nanjing University, \\ 22 Hankou Rd., Nanjing 210093, China
}

(Received 15 May 2012; accepted 5 June 2012; published online 6 July 2012)

\begin{abstract}
Magnetic relaxation is ubiquitous in magnetic materials, and elucidation of the underlying mechanisms is important for achieving reliable device operations. Here, we systematically investigate the magnetic relaxation in compressively strained $\mathrm{La}_{0.7} \mathrm{Sr}_{0.3} \mathrm{MnO}_{3}$ thin films. Upon the removal of external magnetic field, the slow time-dependent increase of in-plane magnetization is correlated with the break-up of magnetic domains and the emergence of additional domain walls, whereas a reduction of magnetization for the initial short period dominates the magnetic relaxation at lower temperatures in thinner films. These relaxation effects underline the importance of domain dynamics in the properties of magnetic thin films. (C) 2012 American Institute of Physics. [http://dx.doi.org/10.1063/1.4733320]
\end{abstract}

External magnetic agitation to a ferromagnetic material can result in deviation of the magnetization vector from its equilibrium position. Upon releasing the external magnetic field, the system tends to relax back to its ground state, a process widely known as magnetic relaxation. The investigation of magnetic relaxation can be traced back to the study of time-dependent magnetic properties of monodomain magnetic particles in 1950s. ${ }^{1,2}$ A thorough understanding of such a relaxation process can be crucial for achieving reliable operation of data storage devices where the stability of the magnetization vector and the distribution of relaxation time must be considered and understood. ${ }^{3}$ Furthermore, the relaxation mechanism can be associated with the time-dependent reconfiguration of magnetic domains, ${ }^{4}$ which may help shed light on the physics behind the domain wall (DW) dynamics in magnetic materials.

Perovskite manganites have attained a lot of attention as these materials exhibit fascinating properties such as half metallicity, colossal magnetoresistance (CMR), and high $\mathrm{Cu}-$ rie temperature $\left(\mathrm{T}_{\mathrm{c}}\right),{ }^{5-7}$ and they also have potential applications in solid oxide fuel cells and resistive switching memory devices. ${ }^{8-11}$ Sirena et al. explored the time dependent physical properties of $\mathrm{La}_{0.6} \mathrm{Sr}_{0.4} \mathrm{MnO}_{3}$ films and suggested that the relaxation process consists of a logarithmic and an exponential terms acting in series, which indicates the presence of both single energy barrier and distributed energy barrier for magnetization switching. ${ }^{12}$ Later, Deac et al. reported both ferromagnetic domain reorientation and phase transformation from ferromagnetic to non-ferromagnetic state as the underlying mechanisms for magnetization and resistivity relaxation in $\mathrm{La}_{0.25} \mathrm{Pr}_{0.375} \mathrm{Ca}_{0.375} \mathrm{MnO}_{3}$ films. ${ }^{13}$ Similar relaxation phenomena were also reported in $\mathrm{La}_{1-\mathrm{x}} \mathrm{Ca}_{\mathrm{x}} \mathrm{MnO}_{3}$ films where domain wall displacement and spin disorder were suggested to be the responsible mechanism. ${ }^{14,15}$

Most of the prior studies on relaxation mechanism in manganites depicted the time dependence of magnetization by interpreting superconducting quantum interference device (SQUID) magnetometer and transport measurement

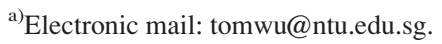

data. ${ }^{12-15}$ However, direct imaging of magnetic domains has been lacking in the literature so far, and there is a need for elucidating the magnetic relaxation process from the microscopic perspective of magnetic domains. Furthermore, there has been no systematic study on the dependence of magnetic relaxation in compressively strained manganite films on the film thickness and measurement temperatures.

Here, by using complementary techniques of magnetic force microscopy (MFM) and SQUID, we investigated the magnetic relaxation in compressively strained $\mathrm{La}_{0.7} \mathrm{Sr}_{0.3} \mathrm{MnO}_{3}$ (LSMO) films from both microscopic and macroscopic perspectives. The results suggest that the dynamic evolution of magnetic domains in manganite films can be correlated with the time dependent data of magnetization and resistance. Furthermore, our data suggest that the behavior of magnetic relaxation in manganite films is quite rich: a fast decrease of magnetization upon the removal of external magnetic field can be related to the rotation of magnetization vector, while the gradual increase afterwards on the time scale of minutes even hours appears to be a result of the slow dynamics of magnetic DWs. Overall, the magnitude of magnetization relaxation in manganite films is a non-monotonous function of film thickness, and the process becomes much slower at low temperatures.

A series of LSMO films with the thickness ranging from 50 to $325 \mathrm{~nm}$ were grown on (100) $\mathrm{LaAlO}_{3}$ (LAO) substrates in a pulsed laser deposition system. The details of film deposition and structural characterization are similar to our previous reports. ${ }^{16-19}$ The magnetic domain of the LSMO films were imaged at room temperature using MFM. ${ }^{20,21}$ The structural studies confirmed that all the LSMO films were coherently grown on the LAO substrates. Because the lattice constant of LAO $(0.378 \mathrm{~nm})$ is smaller than that of LSMO $(0.388 \mathrm{~nm})$, the LSMO films are compressively strained (the detailed thicknessdependent lattice constants can be found in Ref. 20).

Before starting the magnetization and resistance relaxation measurements, a 6000 Oe in-plane magnetic field generated by an electromagnet was applied for $5 \mathrm{~min}$ along the film plane, and then the field was gradually reduced to zero 
$(t=0)$ at a rate of $3000 \mathrm{Oe} / \mathrm{min}$. Imaging a typical area of $1 \mu \mathrm{m} \times 1 \mu \mathrm{m}$ by MFM takes approximately 4 mins. Furthermore, the film surface morphology was checked by atomic force microscopy (AFM) and scanning electron microscopy. The time-dependent magnetization and resistance data were recorded by SQUID and four-probe resistivity measurement setup, respectively. To confirm the accuracy and validity of the magnetization data, we repeated each SQUID measurement several times by using different settling times between data points and found the magnitude of the relaxation to be independent of such measurement parameters.

Figs. 1(a)-1(c) show three typical MFM images taken at the same position on a LSMO film with a thickness of $135 \mathrm{~nm}$ at zero field subsequently at $t=5,70$, and $130 \mathrm{~min}$ after the removal of the external magnetic field. It has been well established that the compressively strained LSMO films with the appropriate thickness exhibit the out-of-plane magnetic domains which can be readily observed by MFM..$^{20,22-24}$ Because the initial applied field is along the $\mathrm{Y}$ direction which is also the (010) crystalline axis determined by the glancing-angle $\mathrm{x}$-ray diffraction experiment, the stripe domains are predominantly aligned along the same direction. After turning off the field, DWs tend to redistribute themselves towards a lower energy state, and this relaxation process can be slow enough to allow the MFM imaging. ${ }^{25}$ The intricate combination of thermal agitation and complex competition between the exchange interaction and the dipoledipole interaction eventually leads the DWs to form mostly perpendicular to the magnetic stripes and break the continuity of the stripe domains. The energy barrier for break-ups of magnetic domains can be quite small. This is similar to the other physical processes in manganites which are characterized by small energy scales, contributing to the complex phase diagrams and magnetic properties of manganites. ${ }^{26}$
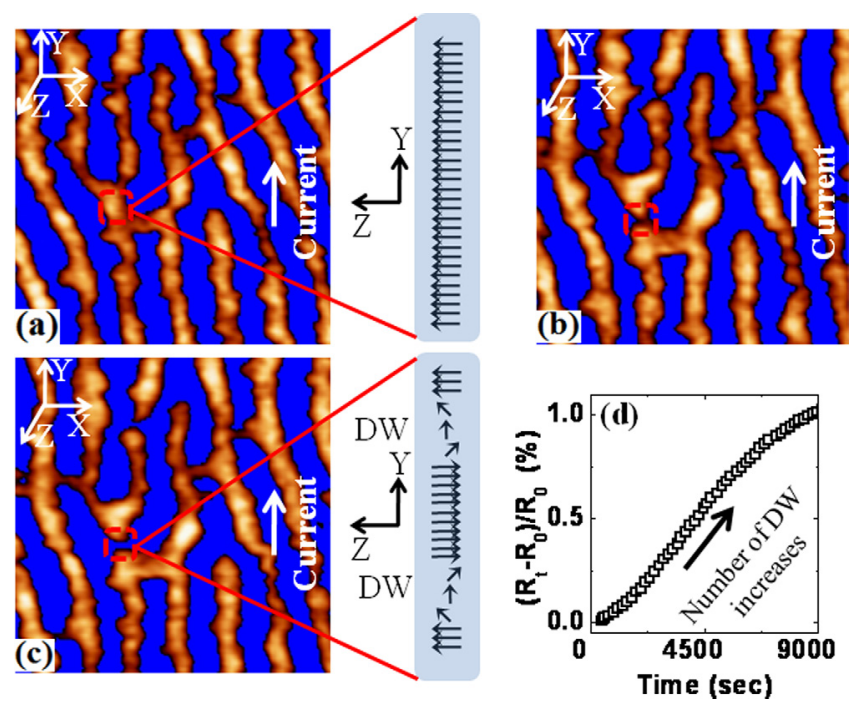

FIG. 1. MFM images (taken at the same place) of the $135 \mathrm{~nm}$ LSMO film at (a) 5, (b) 70, and (c) $130 \mathrm{~min}$ after the external magnetic field (applied along the Y direction) was switched off. The scan size is $1 \mu \mathrm{m} \times 1 \mu \mathrm{m}$. The marked square highlights one particular case of the emergence of additional domain walls. The configurations of the DWs are shown in the schematics on the right side of the MFM images. Note that the coordinates of the schematics are different from those of the MFM images for clarity. (d) Increase of resistance with time as a result of the enhanced scattering of electrons at DWs.
One example of the time-dependent creation of DWs is highlighted in the square area in Figs. 1(a)-1(c).

As the newly created DWs during the relaxation process are observed to align perpendicular to the initial DW direction, to measure the resulting resistance change, we passed the current along the $\mathrm{Y}$ direction and recorded a slow increase in the sample resistance with time. It is well known that the resistivity of DWs in manganites can be several orders larger than that of the traditional 3d transition metals such as Co. ${ }^{27,28} \mathrm{Wu}$ et al. showed that highly resistive DWs in a LSMO film with stripe domains significantly affect the current flow. ${ }^{28}$ Similarly, in our samples, as the current flows in the Y direction, the newly created DWs act as scattering centers for the carriers, resulting in an increase of the overall resistance (Fig. 1(d)).

Now let us focus on the effect of magnetic domain relaxation on the time-dependent magnetization measured in LSMO films (Fig. 2). On the scale of hours, the in-plane magnetization along the $\mathrm{Y}$ axis for all samples increases with time, behaving similarly to the resistance relaxation; it does not saturate within our experimental time window $(2.5 \mathrm{~h})$, suggesting a very slow magnetic evolution process. The enhancement of Y-axis magnetization can be explained if we consider the microscopic structure of the DW. For the films with perpendicular anisotropy and stripe domains, the landscape of DW magnetization can vary locally and the DWs at the film surface is most likely the Néel type. ${ }^{29}$ As shown in Fig. 1(c), the magnetization vector in the Néel wall gradually rotates in the YZ plane, producing a net magnetization component along the $\mathrm{Y}$ axis. As more DWs appear with time, the magnetic moment along $\mathrm{Y}$ direction also

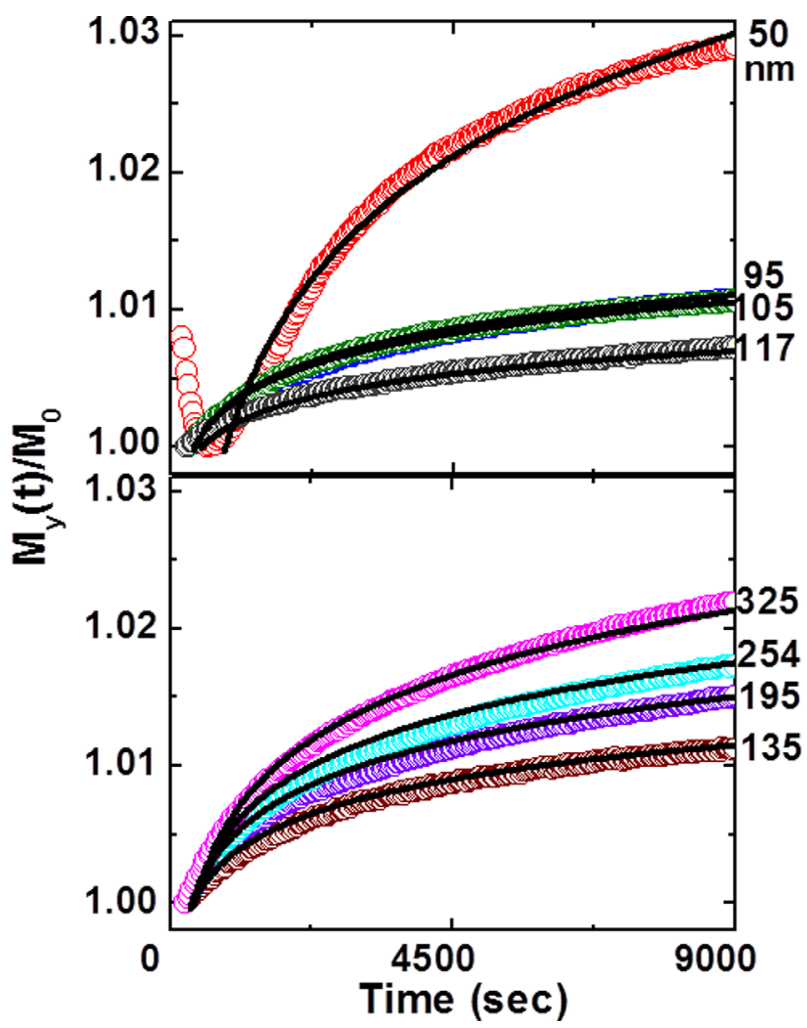

FIG. 2. Time dependence of in-plane magnetization measured at $300 \mathrm{~K}$ for (a) 50, 95, 105, and $117 \mathrm{~nm}$ and (b) $135,195,254$, and $325 \mathrm{~nm}$ LSMO films. The solid lines are logarithmic fittings to the magnetization relaxation data. 
increases. The magnetization relaxation data can be wellfitted with the logarithmic model $M(t)=M_{0}+S \cdot \ln (t)$, where $M_{o}$ and $S$ are the initial magnetization and the coefficient of magnetic viscosity, respectively. ${ }^{30-32}$ This implies that instead of a single energy barrier, a distribution of the barrier energy is more appropriate to describe the thermally activated magnetization evolution in the LSMO films. ${ }^{33}$

An interesting feature of the time-dependent magnetization data shown in Fig. 2 is the initial reduction of the magnetization over a short time period $(\sim 120 \mathrm{~s})$ observed in the $50 \mathrm{~nm}$ LSMO film. Such an effect has not been reported in manganite thin films before. This initial decrease of magnetization is more prominent in the $50 \mathrm{~nm}$ sample than the thicker ones. This behavior can be explained by the immediate formation of magnetic domains with out-of-plane magnetization upon the removal of applied magnetic field. Due to the compressive-strain-induced perpendicular anisotropy, the local magnetization in domains quickly rotates from the in-plane to the out-of-plane direction, causing an initial reduction of the magnetization in the in-plane $\mathrm{Y}$ direction. For the thinnest sample, the shape anisotropy, which favors the magnetization to lie in-plane, is the strongest. This inplane anisotropy may slow down the initial rotation of the magnetization vector in the thinner films, making the phenomenon observable. On the other hand, this rotation of magnetization can be much faster in the thicker films as a result of weaker magnetic anisotropy, which makes this initial change of magnetization invisible in the "slow" SQUID measurements.

In previous studies, magnetic relaxation in LSMO films was reported to be more prominent in thinner samples than the thicker counterparts. ${ }^{12}$ Indeed, the thinnest LSMO film $(50 \mathrm{~nm})$ exhibits the most significant magnetic relaxation according to our SQUID results (Fig. 2(a)); an increase of $2.95 \%$ in magnetization was observed during the SQUID measurement of $150 \mathrm{~min}$. But, overall our measurements clearly show that the magnitude of the magnetization relaxation is a non-monotonous function of thickness. The ratio $M(t=150 \mathrm{~min}) / M_{0}$ decreases initially with the increasing film thickness up to $117 \mathrm{~nm}$, but the trend reverses above $135 \mathrm{~nm}$ (Fig. 3(a)). As we discuss in the following, such a non-monotonic behavior could be a manifestation of two competing factors, i.e., the energy barrier height for the DW motion and the number of created DWs.

The energy barrier for the DW pinning is correlated to the grain size: the geometric boundaries between grains can work effectively as pinning centers for magnetic domains. By performing the fast Fourier transformation on the AFM images (insets in Fig. 3(b) are the images taken on the 50 and $325 \mathrm{~nm}$ samples) and analyzing the power spectral density of the transformed images, we determined the average grain size for LSMO films with different thicknesses. As shown in Fig. 3(b), the grain size gradually increases from $28 \mathrm{~nm}$ to $67 \mathrm{~nm}$ as the film thickness increases from 50 to $325 \mathrm{~nm}$. The micromagnetic theory suggests that above the exchange length $L_{0}=\sqrt{A / K}$, the coercivity scales inversely with the grain size. ${ }^{34}$ For LSMO, using the standard parameters found in literature, ${ }^{35,36}$ i.e., the exchange stiffness $A=1.73 \times 10^{-12} \mathrm{~J} / \mathrm{m}$ and the anisotropy constant $K=8.4$ $\times 10^{3} \mathrm{~J} / \mathrm{m}^{3}$, this threshold thickness is estimated to be

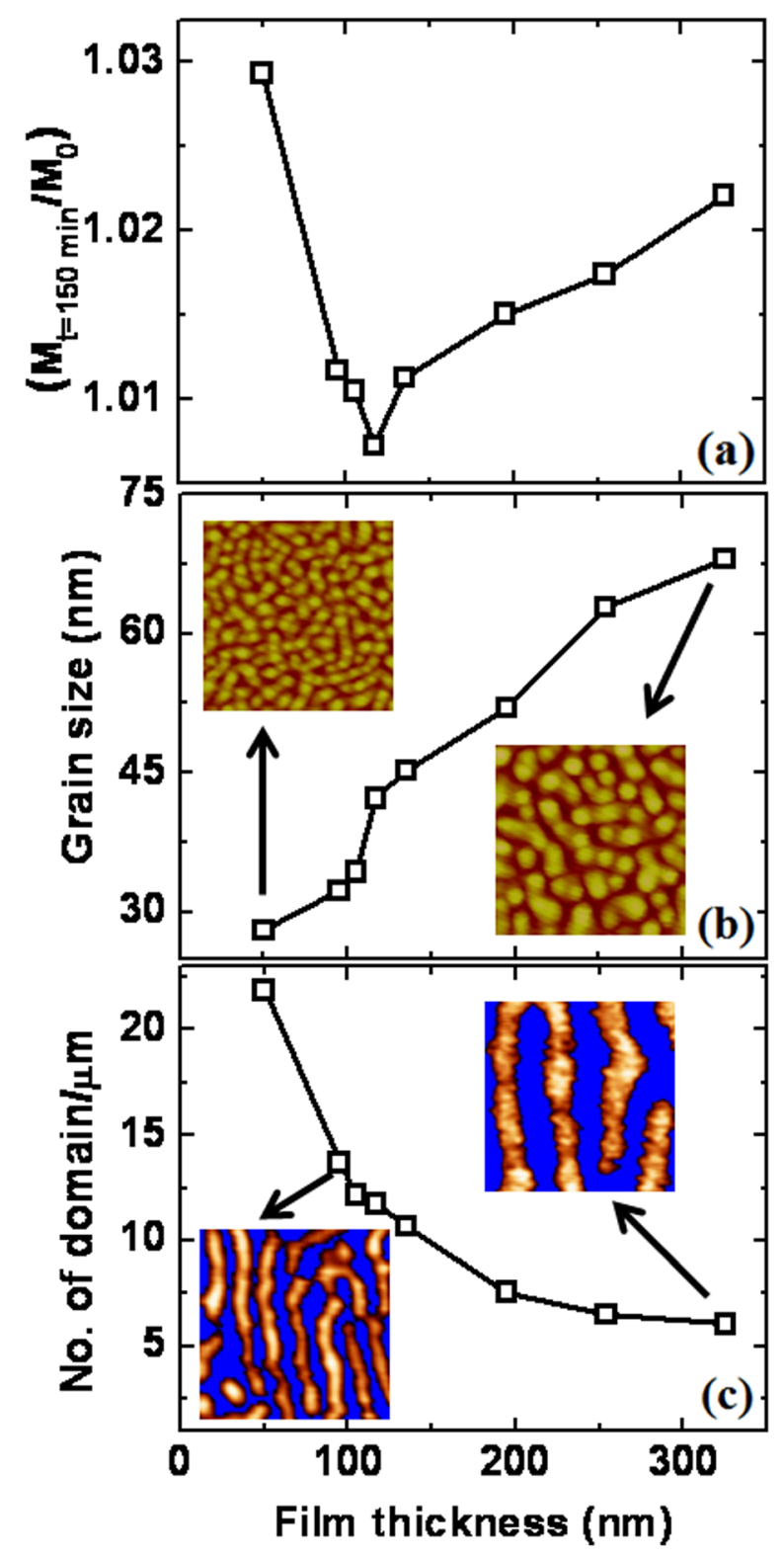

FIG. 3. (a) Thickness dependence of the total change of magnetization in LSMO films measured over $150 \mathrm{~min}$. (b) and (c) show the thickness dependence of the average grain size and the number of magnetic domains per unit length along the $X$ axis (same coordinates as in Fig. 1). The insets in (b) and (c) show the typical AFM and MFM images, respectively. The scan size is $1 \mu \mathrm{m} \times 1 \mu \mathrm{m}$ for all images.

approximately $14 \mathrm{~nm}$, which is smaller than the thickness range studied in this work. In line with the theory, the reduction of coercivity on increasing film thickness has also been observed in other works. ${ }^{20,37}$ Thus, the average energy barrier for the DW movements in LSMO films reduces with the increasing film thickness, which in turn is responsible for the increase in the general domain activities; the possible creation of more domain walls in the thicker LSMO films is consistent with the observation of stronger magnetic relaxation in the films with a thickness larger than $135 \mathrm{~nm}$.

On the other hand, the domains in the LSMO films become larger as the film thickness increases, i.e., the number of stripe domains per unit length along the $\mathrm{X}$ direction (according to the coordinates in Fig. 1) decreases, implying a reduction of the total number of the newly created DWs during the magnetic relaxation process. Fig. 3(c) shows the data 

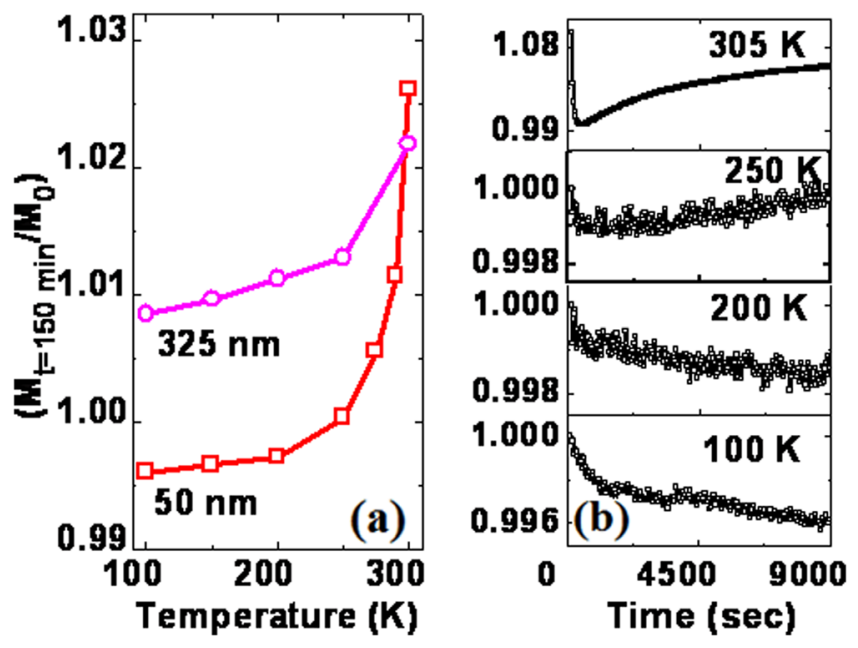

FIG. 4. (a) Temperature dependence of the total magnetization relaxation measured in 50 and $325 \mathrm{~nm}$ LSMO films. (b) Time dependence of magnetization of the $50 \mathrm{~nm}$ sample at different temperatures.

of average number of DWs per $\mu \mathrm{m}$ vs. the film thickness, which suggests that such a thickness-dependent effect is more significant for the samples in the lower thickness range. Reduction of the film thickness from $135 \mathrm{~nm}$ to $50 \mathrm{~nm}$ leads to the appearance of approximately 13 more domains per $\mu \mathrm{m}$, whereas the thickness reduction from $325 \mathrm{~nm}$ to $135 \mathrm{~nm}$ adds only 5 additional domains. This increase in the number of DWs is consistent with the observation of enhanced magnetic relaxation in the films thinner than $117 \mathrm{~nm}$. The combination of the two thickness-dependent factors, i.e., the number of DWs and the variation of the energy barrier for DW motions, likely leads to the observed non-monotonous thickness dependence of the magnetization relaxation in LSMO films (Fig. 3(a)).

To acquire further insights on the thickness dependence of the magnetic relaxation mechanism in LSMO films, we measured time-dependent magnetization at different temperatures. Fig. 4(a) shows the temperature dependence of the total magnetization relaxation, $M(t=150 \mathrm{~min}) / M_{0}$, measured in the $50 \mathrm{~nm}$ and $325 \mathrm{~nm}$ LSMO films. The magnitude of magnetic relaxation quickly drops as the temperature goes down, suggesting a thermal activation mechanism of the DW movement. As the temperature reduces, the DWs become more "frozen" due to the less available thermal energy, causing a lower attempt frequency and a smaller probability for creating DWs. This leads to the reduction of total relaxation in the LSMO films as the measurement temperature goes down.

Furthermore, the temperature dependence of the magnetization relaxation in the $50 \mathrm{~nm}$ sample exhibits a surprising effect. As mentioned earlier, the room temperature magnetization of this thin film reduces initially for a very short period of time (within $2 \mathrm{~min}$ ) before the onset of magnetization increase over the longer time scale (Fig. 2(a)). Interestingly, this time-dependent decreasing trend of magnetization becomes more significant at temperatures below $250 \mathrm{~K}$ (Fig. 4(b)). This could be understood as the result of the stronger in-plane shape anisotropy of thinner LSMO films than that of the thicker ones, and the rotation of magnetic vectors as well as the thermally activated creation of DWs becomes very slow at low temperatures.
In summary, our results revealed rich phenomena of magnetic relaxation in compressively strained ferromagnetic manganite films. After removal of applied magnetic field, we directly imaged the time-dependent evolution of magnetic domains at zero-field using room temperature MFM, and the creation of additional DWs can be readily correlated with the observed magnetic relaxation in the SQUID measurements. These results not only underline the importance of the time-dependent aspect in the magnetic measurements but also motivate further studies on the dynamic characteristics of magnetic domains in ferromagnetic oxide thin films.

${ }^{1}$ C. P. Bean and J. D. Livingstone, J. Appl. Phys. 30, S120 (1959).

${ }^{2}$ L. Néel, J. Phys. Radium 11, 49 (1950).

${ }^{3}$ A. Lyberatos, R. W. Chantrell, E. R. Sterringa, and J. C. Lodder, J. Appl. Phys. 70, 4431 (1991).

${ }^{4}$ L. C. Sampaio, M. P. de Albuquerque, and F. S. de Menezes, Phys. Rev. B 54, 6465 (1996).

${ }^{5}$ J.-H. Park, E. Vescovo, H.-J. Kim, C. Kwon, R. Ramesh, and T. Venkatesan, Phys. Rev. Lett. 81, 1953 (1998).

${ }^{6}$ M. Ziese, Rep. Prog. Phys. 65, 143 (2002).

${ }^{7}$ Y. Tokura, Rep. Prog. Phys. 69, 797 (2006).

${ }^{8}$ S. P. Jiang, J. Mater. Sci. 43, 6799 (2008).

${ }^{9}$ C. Sun, R. Hui, and J. Roller, J. Solid State Electrochem. 14, 1125 (2010).

${ }^{10}$ Y. B. Nian, J. Strozier, N. J. Wu, X. Chen, and A. Ignatiev, Phys. Rev. Lett. 98, 146403 (2007).

${ }^{11}$ S. Asanuma, H. Akoh, H. Yamada, and A. Sawa, Phys. Rev. B 80, 235113 (2009).

${ }^{12}$ M. Sirena, L. B. Steren, and J. Guimpel, Phys. Rev. B 64, 104409 (2001).

${ }^{13}$ I. G. Deac, S. V. Diaz, B. G. Kim, S.-W. Cheong, and P. Schiffer, Phys. Rev. B 65, 174426 (2002).

${ }^{14}$ R. von Helmolt, J. Wecker, T. Lorenz, and K. Samwer, Appl. Phys. Lett. 67, 2093 (1995).

${ }^{15}$ M. Ziese, S. P. Sena, and H. J. Blythe, J. Magn. Magn. Mater. 202, 292 (1999).

${ }^{16}$ Y. G. Zhao, R. C. Srivastava, P. Fournier, V. Smolyaninova, M. Rajeswari, T. Wu, Z. Y. Li, R. L. Greene, and T. Venkatesan, J. Magn. Magn. Mater. 220, 161 (2000).

${ }^{17}$ T. Wu and J. F. Mitchell, Phys. Rev. B 69, R100405 (2004).

${ }^{18}$ T. Wu and J. F. Mitchell, Appl. Phys. Lett. 86, 252505 (2005).

${ }^{19}$ J. F. Ding, K. X. Jin, Z. Zhang, and T. Wu, Appl. Phys. Lett. 100, 062402 (2012).

${ }^{20}$ S. R. Bakaul, W. Lin, and T. Wu, Appl. Phys. Lett. 99, 042503 (2011).

${ }^{21}$ S. R. Bakaul, W. Lin, and T. Wu, Appl. Phys. Lett. 100, 012403 (2012).

${ }^{22}$ C. Kwon, M. C. Robson, K.-C. Kim, J. Y. Gu, S. E. Lofland, S. M. Bhagat, Z. Trajanovic, M. Rajeswari, T. Venkatesan, A. R. Kratz, R. D. Gomez, and R. Ramesh, J. Magn. Magn. Mater. 172, 229 (1997).

${ }^{23}$ J. Dho, Y. N. Kim, Y. S. Hwang, J. C. Kim, and N. H. Hur, Appl. Phys. Lett. 82, 1434 (2003).

${ }^{24}$ J. Dho and N. H. Hur, J. Magn. Magn. Mater. 318, 23 (2007).

${ }^{25}$ See supplementary material at http://dx.doi.org/10.1063/1.4733320 for a movie made of consecutive MFM images collected within about five hours.

${ }^{26}$ E. Dagotto, Science 309, 257 (2005).

${ }^{27}$ N. D. Mathur, P. B. Littlewood, N. K. Todd, S. P. Isaac, B.-S. Teo, D.-J. Kang, E. J. Tarte, Z. H. Barber, J. E. Evetts, and M. G. Blamire, J. Appl. Phys. 86, 6287 (1999).

${ }^{28}$ Y. Wu, Y. Suzuki, U. Rudiger, J. Yu, A. D. Kent, T. K. Nath, and C. B. Eom, Appl. Phys. Lett. 75, 2295 (1999).

${ }^{29}$ A. Hubert and R. Schaefer, Magnetic Domains (Springer, New York, 1998).

${ }^{30}$ C. N. Guy, J. Phys. F: Met. Phys. 8, 1309 (1978).

${ }^{31}$ E. P. Wohlfarth, J. Phys. F: Met. Phys. 14, L155 (1984).

${ }^{32}$ L. M. Fisher, A. V. Kalinov, S. E. Savel'ev, I. F. Voloshin, and A. M. Balbashov, J. Phys.: Condens. Matter 10, 9769 (1998).

${ }^{33}$ P. Gaunt, J. Appl. Phys. 59, 4129 (1986).

${ }^{34}$ G. Herzer, IEEE Trans. Magn. 26, 1397 (1990).

${ }^{35}$ R. Akiyama, H. Tanaka, T. Matsumoto, and T. Kawai, Appl. Phys. Lett. 79, 4378 (2001).

${ }^{36}$ K. Steenbeck, T. Habisreuther, C. Dubourdieu, and J. P. Sénateur, Appl. Phys. Lett. 80, 3361 (2002).

${ }^{37}$ S. Roy, I. Dubenko, D. D. Edorh, and N. Ali, J. Appl. Phys. 96, 1202 (2004). 